\title{
A new analgesic paradigm for pediatric tonsillectomy: Are we dragging our heels?
}

\author{
Mohamad Ahmad, MD • David Sommerfreund, MD
}

Received: 9 September 2015/Revised: 31 October 2015/Accepted: 3 December 2015/Published online: 15 December 2015

(c) Canadian Anesthesiologists' Society 2015

In pediatrics, postoperative analgesic options are relatively limited and the barriers to optimal analgesia are numerous. Currently available analgesics have various undesirable side effects, including sedation, respiratory depression, or interference with coagulation. In addition, various regulatory, scientific, industry, physician, and parental barriers exist.

Arguably, there is no greater need for better analgesia than among children undergoing tonsillectomy or adenotonsillectomy. The pain of tonsillectomy lasts longer than most other surgeries-four to seven days ${ }^{1}$ with residual pain on swallowing often lasting longer. The very serious risk of postoperative hemorrhage is traditionally the foremost concern after tonsillectomy and occurs in approximately $3 \%$ of patients. ${ }^{2}$ In comparison, significant pain after inguinal hernia repair and other common pediatric surgeries typically lasts only one to two days. In such cases, children can return to a normal routine by three days ${ }^{1}$ and bleeding is a negligible concern.

In this issue of the Journal, Li et al. ${ }^{3}$ from Sichuan, China make a significant contribution to the literature with a novel report on the efficacy of parecoxib in tonsillectomy and adenotonsillectomy. Parecoxib is a prodrug of orally-administered valdecoxib (Bextra; GD Searle \& Company, New York, NY, USA) that is administered intravenously. Parecoxib and valdecoxib are selective cyclooxygenase-2 (COX-2) inhibitors. Drugs of this class reduce the production of inflammation-promoting prostaglandins while, unlike nonselective nonsteroidal

M. Ahmad, MD $(\varangle) \cdot$ D. Sommerfreund, MD Department of Anesthesia and Perioperative Medicine, Western University, Victoria Campus C2-168, 800 Commissioners Road East, London, ON N6A 5W9, Canada

e-mail: mohamad.ahmad@lhsc.on.ca anti-inflammatory drugs (NSAIDs), spare prostaglandins maintain platelet aggregation, bronchial tone, and gastric mucosal integrity. Thus far, an association between neurotoxicity, an increasingly important consideration in pediatric anesthesia, and NSAIDs or COX-2 inhibitors is lacking in the published literature.

Cyclooxygenase- 2 inhibitors do increase the risk of cardiovascular atherothrombosis, such as myocardial infarction and stroke in adults, ${ }^{4}$ and for this reason, they were largely withdrawn from the North American market in 2004 (rofecoxib) and 2005 (valdecoxib). Indeed, Health Canada specifically discourages the short-term and perioperative use of valdecoxib. Parecoxib has never been approved by Health Canada or the United States Food and Drug Administration (FDA), although it is widely available in many other countries. Atherothrombotic risk in children is presumably low, but it is unrealistic to expect a large study to be conducted to confirm that assumption. For a variety of reasons, few drug approval studies are conducted on pediatric patients, the relatively small size of the pediatric drug market being one of them. As a result, most drugs are simply used "off label" in children. With no adult market to serve and to drive the relevant regulatory pathways, parecoxib will likely remain unavailable in North America.

Li et al. studied 60 children who were undergoing tonsillectomy or adenotonsillectomy and randomized to receive either parecoxib $1 \mathrm{mg} \cdot \mathrm{kg}^{-1}$ or saline at induction of anesthesia. The authors found that the saline group had significantly worse median Children's Hospital of Eastern Ontario Pain Scale pain scores (placebo, 9 [8-11] vs parecoxib, 7 [5-8]; $P<0.001)$ in the postanesthesia care unit (PACU), needed more analgesic rescues with morphine (relative risk $[\mathrm{RR}], 1.5 ; 95 \%$ confidence interval $[\mathrm{CI}], 1.0$ to $2.1 ; P=0.024)$, needed rescue 
analgesics $61 \mathrm{~min}$ earlier (95\% CI, 27 to $91 ; P=0.001$ ), and consumed $53 \%$ more overall morphine $(P=0.017)$. In addition, the saline group had more postoperative nausea and sedation (RR, 2.8; 95\% CI, 1.0 to $7.7 ; P=0.037$ ). There were no differences in pain scores after discharge from the PACU, airway problems, or the incidence of bleeding in this study, although it was not powered to detect differences for these complications. There were no reported atherothrombotic events.

Overall, the study was well designed, but there were some limitations, such as the one-time dosing of parecoxib and a relatively short $24-\mathrm{hr}$ follow-up period. Postoperative nausea and vomiting prophylaxis was atypical in that dexamethasone dosing was high $\left(0.5 \mathrm{mg} \cdot \mathrm{kg}^{-1}\right)$ and prophylactic ondansetron was not administered at all.

In the July 2015 issue of the Journal, Murto et al. ${ }^{5}$ published a similar and important study using celecoxib (also a COX-2 inhibitor) for the treatment of tonsillectomy pain in children ages two to $18 \mathrm{yr}$. Celecoxib is available in North America but not as an intravenous formulation. Children in the treatment group received $6 \mathrm{mg} \cdot \mathrm{kg}^{-1}$ of celecoxib orally preoperatively followed by $3 \mathrm{mg} \cdot \mathrm{kg}^{-1}$ twice daily for five doses. The doses were chosen by extrapolating adult data, although celecoxib clearance may be higher in children. ${ }^{6}$ Study findings showed an $11 \%$ reduction in visual analogue pain scores over the first two postoperative days $(P=0.04)$. The incidence of adverse events and bleeding was also similar between the groups. In contrast to Murto et al., there was no overall reduction in opioid consumption reported in the present Li et al. study. The incidence of adverse events and bleeding was similar between the studies.

Interestingly, patient genotyping was also undertaken in the Murto study. Those who were heterozygous for the CYP2C9*3 allele, an indicator of reduced celecoxib metabolism, had better analgesia for seven days postoperatively. Those without the allele had worse analgesia than placebo after the second day. This may indicate that the celecoxib dose was too low. The optimal dose of celecoxib is unknown because basic pharmacologic data are insufficient. When Murto et al. initiated their study, the only available pharmacokinetic study of celecoxib in children was conducted in ten oncology patients (aged seven years or older). ${ }^{6}$ This earlier study showed higher celecoxib clearance and shorter half-life in children compared with adults. A second pharmacokinetic study ${ }^{7}$ was published in 2012, just as they were concluding their study. Despite FDA approval for celecoxib in 1998, even today, the appropriate dose of celecoxib in children is unknown. Indeed, the recommended dose is imprecise and varies more than twofold. ${ }^{7}$ Two pediatric studies over 17 years is evidently insufficient.

Li $e t$ al. and Murto et al. both add to a growing body of publications over the last five years calling for a paradigm shift in how we treat the pain and side effects of tonsillectomy. The impetus is twofold. First, the indications for tonsillectomy and adenotonsillectomy in North America increasingly focus on the treatment of sleep-disordered breathing and obstructive sleep apnea (OSA) rather than on the traditional indication of recurrent tonsillitis. ${ }^{8}$ The second reason relates to the recognition that children with OSA are at especially high risk for opioid-induced postoperative respiratory depression..$^{9,10}$ Furthermore, a disproportionately high number of post-tonsillectomy deaths and neurological injuries in children with OSA are due to primary apneic causes rather than to hemorrhage, which was the predominant cause in children without OSA. ${ }^{11}$ Clearly, a non-opioid analgesic is needed for future tonsillectomies.

The underutilization of NSAIDS after tonsillectomy is largely because of persistent concerns about bleeding. The platelet-sparing effects of COX-2 inhibitors are well established. ${ }^{12}$ Furthermore, the balance of research indicates that even for ibuprofen, a nonselective NSAID, does not increase post-tonsillectomy bleeding and is safe. ${ }^{13}$ A recent meta-analysis involving over 1,747 children supports this view. ${ }^{14}$ In contrast, ketorolac, which is much less COX-2 selective, is not safe. ${ }^{13}$ Indeed, Ontario's 2013 tonsillectomy guidelines recommend routine ibuprofen for tonsillectomy but warn against use of ketorolac. ${ }^{15}$

In a recent study comparing morphine with ibuprofen after tonsillectomy, Kelly et al. provide compelling evidence for the routine administration of ibuprofen. ${ }^{16}$ In their study, 91 pediatric patients with sleep-disordered breathing were randomized to perioperative ibuprofen or morphine and had overnight oximetry pre- and postoperatively. During the first postoperative night, oxygen desaturation improved in 68\% of the ibuprofen group but only in $14 \%$ of the morphine group $(P<0.01)$. Furthermore, the number of desaturation episodes per hour decreased in the ibuprofen group but increased in the morphine group - so much so that the study was stopped early by the study's Data Safety Monitoring Board. The average duration of analgesic use (either morphine or ibuprofen) was approximately four days in both groups. Both groups also had similar pain scores despite the relatively high dose of morphine prescribed (0.2-0.5 $\mathrm{mg} \cdot \mathrm{kg}^{-1}$ po every four hours as needed). There was no difference in postoperative bleeding, but the study was also underpowered to address this complication.

We do not know the current rate of ibuprofen use for tonsillectomy-anecdotally, it seems to be low. Can the rate be improved? The adoption rate of routine dexamethasone, which is promoted by evidence-based American guidelines for postoperative nausea and vomiting after tonsillectomy, may provide some insight into barriers to adoption. In 2011, the American Academy 
of Otolaryngology-Head and Neck Surgery proposed evidence-based guidelines for the management of adenotonsillectomy. ${ }^{13}$ It did not address postoperative analgesia directly but did advocate for the routine use of dexamethasone and against the routine use of antibiotics. Nevertheless, in a review of 111,813 tonsillectomies and adenotonsillectomies published in 2015, Mahant et al. reported that dexamethasone use increased only marginally-from $74.6 \%$ of procedures prior to the guidelines to $77.4 \%$ of procedures in $2013(P<0.001){ }^{2}$ This may appear to be a good response, but by comparison, would we accept an automobile that was only $77.4 \%$ reliable? Importantly, these were aggregate statistics for all patients; the range of adoption within individual hospitals varied from 63.3-95.7\% (i.e., one-third of patients did not receive any dexamethasone in some hospitals). Conversely, antibiotic use had decreased (as recommended) much more significantly from 34.7 to $21.8 \%$ ( $P<0.001$ ). The authors discuss a number of potential reasons why dexamethasone use did not improve, including the discredited but persistent belief based on one well-publicized study from 2008 that dexamethasone increases postoperative bleeding. ${ }^{17}$ That 2008 study was an outlier in the dexamethasone literature, and its statistical analyses have been extensively questioned. ${ }^{18}$ Ibuprofen and other nonselective NSAIDs face precisely the same challenge if a bleeding risk rationale-however unproven-is also considered to be true.

In light of this instructive example, and despite guidelines $^{13,15}$ endorsing it, we cannot realistically expect ibuprofen to be universally adopted by physicians anytime soon. Its association with postoperative bleeding may be too deeply ingrained in the collective pediatric consciousness. Perhaps we need a clean start with a new class of analgesic drugs such as COX-2 inhibitors. More study is needed. Nevertheless, with all the other barriers to optimal pediatric analgesia, physician complacency should not be one of them.

\section{Un nouveau paradigme analgésique pour les amygdalectomies pédiatriques: sommes-nous à la traîne?}

En pédiatrie, les options d'analgésie postopératoire sont relativement limitées et les obstacles à une analgésie optimale sont nombreux. Les analgésiques disponibles à l'heure actuelle s'accompagnent de divers effets secondaires indésirables, dont la sédation et la dépression respiratoire, et ils peuvent également interférer avec la coagulation. À cela s'ajoutent d'autres obstacles d'ordre légal ou scientifique, ou encore liés à l'industrie, aux médecins et aux parents.

Il ne fait aucun doute que le besoin pour une meilleure analgésie est plus pressant que jamais pour les enfants subissant une amygdalectomie ou une adéno-amygdalectomie. La douleur liée à l'amygdalectomie dure de quatre à sept jours, ${ }^{1}$ soit plus longtemps que celle découlant de la plupart des chirurgies, et une douleur résiduelle se fait souvent ressentir encore plus longtemps lors de la déglutition. L'inquiétude première après une amygdalectomie est en général liée au risque très sérieux d'hémorragie postopératoire; cette complication survient chez environ $3 \%$ des patients. ${ }^{2} \grave{A}$ titre de comparaison, la douleur prononcée ne dure généralement qu'un ou deux jours après une réparation d'hernie inguinale ou toute autre chirurgie pédiatrique courante. Dans ces cas-là, les enfants peuvent reprendre leur routine trois jours plus tard ${ }^{1}$ et les saignements ne sont qu'une préoccupation mineure.

Dans ce numéro du Journal, Li et coll., des chercheurs de la province du Sichuan, en Chine, présentent une importante contribution à la littérature en publiant un compte rendu innovant sur l'efficacité du parécoxib dans les cas d'amygdalectomie et d'adéno-amygdalectomie. Le parécoxib qu'on administre par voie intraveineuse est un promédicament de valdécoxib, un agent administré oralement (Bextra; GD Searle \& Company, New York, NY, États-Unis). Le parécoxib et le valdécoxib sont des inhibiteurs sélectifs de la cyclo-oxygénase-2 (COX-2). Les médicaments de cette classe, à l'inverse des anti-inflammatoires non stéroïdiens (AINS) non sélectifs, réduisent la production de prostaglandines pro-inflammatoires mais épargnent l'agrégation plaquettaire, le tonus bronchique et l'intégrité de la muqueuse gastrique. À ce jour, la littérature publiée n'a pas montré d'association entre une quelconque neurotoxicité-une préoccupation de plus en plus importante en anesthésie pédiatrique—et les AINS ou les inhibiteurs de la COX-2.

Nous savons que, chez l'adulte, les inhibiteurs de la cyclo-oxygénase 2 augmentent le risque d'athérothrombose, comme par exemple les infarctus du myocarde et les accidents vasculaires cérébraux. ${ }^{4} \mathrm{C}$ 'est pour cette raison que ces agents ont été, pour la plupart, retirés du marché nord-américain en 2004 (rofécoxib) et 2005 (valdécoxib). En effet, Santé Canada déconseille spécifiquement l'usage à court terme et périopératoire du valdécoxib. Quant au parécoxib, cet agent n'a jamais été approuvé par Santé Canada ni par la FDA (Food and Drug Administration) américaine, bien qu'il soit disponible dans bon nombre d'autres pays. Selon toute vraisemblance, le risque d'athérothrombose est bas chez l'enfant. Ceci étant dit, il est très peu probable qu'une étude d'envergure soit jamais menée pour confirmer cette 
hypothèse. Pour diverses raisons, très peu d'études d'approbation de médicaments sont réalisées chez des patients pédiatriques, la taille relativement restreinte de ce marché en étant une. Par conséquent, la plupart des médicaments sont tout simplement utilisés « hors indication » chez l'enfant. Comme il n'y a pas de marché à desservir chez l'adulte, ce qui motiverait les démarches règlementaires pertinentes jusqu'à l'autorisation d'utiliser cet agent, il est probable que le parécoxib ne devienne jamais disponible en Amérique du Nord.

L'étude de Li et coll. a porté sur 60 enfants subissant une amygdalectomie ou une adéno-amygdalectomie qui ont été aléatoirement répartis à recevoir soit $1 \mathrm{mg} \cdot \mathrm{kg}^{-1} \mathrm{de}$ parécoxib, soit une solution physiologique à l'induction de l'anesthésie. Les auteurs ont observé que les enfants du groupe recevant la solution physiologique affichaient des scores de douleur moyens significativement plus élevés sur l'Échelle de douleur de l'hôpital (placebo, 9 [8-11] vs parécoxib, 7 [5-8]; $P<0,001)$ en salle de réveil, avaient besoin de plus d'analgésie de secours avec morphine (risque relatif [RR], 1,5; intervalle de confiance [IC] $95 \%$, $1,0$ à 2,1; $P=0,024)$, avaient besoin d'analgésie de secours 61 minutes plus tôt (IC $95 \%, 27$ à 91; $P=0,001)$ et avaient consommé globalement $53 \%$ plus de morphine $(P=$ 0,017). De plus, le groupe solution physiologique a davantage souffert de nausées et de sédation postopératoires (RR, 2,8; IC $95 \%, 1,0$ à 7,7; $P=0,037$ ). Dans cette étude, aucune différence n'a été observée en matière de scores de douleur après le congé de la salle de réveil, de complications au niveau des voies aériennes, ou d'incidence de saignement, bien que l'étude ait eu suffisamment de puissance pour détecter toute différence pour ces complications. Aucun cas d'athérothrombose n'a été rapporté.

D’une manière générale, l'étude était bien conçue, mais elle comportait certaines limites, comme par exemple l'administration d'une dose unique de parécoxib et une période de suivi relativement courte de $24 \mathrm{~h}$. La prophylaxie pour prendre en charge les nausées et vomissements postopératoires était atypique : le dosage de dexaméthasone était élevé $\left(0,5 \mathrm{mg} \cdot \mathrm{kg}^{-1}\right)$ et aucun ondansétron prophylactique n'a été administré.

Dans le numéro de juillet 2015 du Journal, Murto et coll. $^{5}$ ont quant à eux publié une autre étude d'importance semblable qui évaluait l'efficacité du célécoxib (un autre inhibiteur de la $\mathrm{COX}-2$ ) pour traiter la douleur liée à l'amygdalectomie chez des enfants âgés de deux à 18 ans. Le célécoxib est disponible en Amérique du Nord, mais pas en formulation intraveineuse. Les enfants $\mathrm{du}$ groupe recevant le traitement ont reçu $6 \mathrm{mg} \cdot \mathrm{kg}^{-1}$ de célécoxib par voie orale en période préopératoire, puis $3 \mathrm{mg} \cdot \mathrm{kg}^{-1}$ deux fois par jour pour un total de cinq doses. Dans cette étude, les doses ont été choisies en extrapolant des données pertinentes à l'adulte, bien que l'élimination du célécoxib soit peut-être plus élevée chez l'enfant. ${ }^{6}$ Les résultats de l'étude ont montré une réduction de $11 \%$ sur les échelles de douleur visuelles analogiques au cours des deux premiers jours postopératoires $(P=0,04)$. L'incidence d'effets néfastes et de saignements était également semblable dans les deux groupes. Contrairement à l'étude de Murto et coll., aucune réduction globale de la consommation d'opioïdes n'a été rapportée dans l'étude de Li et coll., publiée dans ce numéro. L'incidence d'effets néfastes et de saignements était semblable dans les deux études.

Fait intéressant, le génotypage des patients a également été entrepris dans l'étude de Murto, et les patients hétérozygotes au niveau de l'allège CYP2C9*3, un indicateur de métabolisme réduit du célécoxib, ont bénéficié d'une meilleure analgésie pendant sept jours postopératoires. L'analgésie des patients ne possédant pas cet allèle était moins bonne que dans le groupe placebo après le deuxième jour. Cette observation pourrait indiquer que la dose de célécoxib était trop faible. À ce jour, la dose optimale de célécoxib n'a pas encore été déterminée, parce que nous ne disposons pas de suffisamment de données pharmacologiques de base. Lorsque Murto et coll. ont commencé leurs travaux, la seule étude pharmacocinétique publiée portant sur l'utilisation de célécoxib chez l'enfant avait été menée auprès de dix patients d'oncologie (âgés de sept ans ou plus). ${ }^{6}$ Cette étude montrait une meilleure élimination et une demi-vie plus courte du célécoxib chez l'enfant que chez l'adulte. Une deuxième étude pharmacocinétique $^{7}$ a été publiée en 2012, alors que les auteurs concluaient la leur. Malgré l'approbation accordée par la FDA pour le célécoxib en 1998, la dose adaptée de cet agent chez l'enfant demeure inconnue à ce jour. En fait, la dose recommandée est peu précise et varie du simple au double. ${ }^{7}$ Il est évident que deux études pédiatriques sur 17 ans ne suffisent pas.

Les études de Li et coll. et de Murto et coll. s'ajoutent toutes deux à un corpus grandissant de publications, qui depuis les cinq dernières années, préconisent un changement de paradigme dans la façon dont nous traitons la douleur et les effets secondaires de l'amygdalectomie. Cet élan a deux facettes. Premièrement, en Amérique du Nord, les indications pour l'amygdalectomie et l'adéno-amygdalectomie se concentrent de plus en plus sur le traitement des troubles respiratoires du sommeil et de l'apnée obstructive du sommeil (AOS) plutôt que sur l'indication conventionnelle d'amygdalite récurrente. ${ }^{8}$ La deuxième raison est liée à la prise de conscience que les enfants atteints d'AOS courent un risque particulièrement élevé de dépression respiratoire postopératoire induite par les opioïdes. ${ }^{9,10}$ De plus, un nombre disproportionné de décès post-amygdalectomies et 
de lésions neurologiques chez les enfants atteints d'AOS sont dus à des causes apnéiques primaires plutôt qu'à une hémorragie, la cause prédominante chez les enfants sans AOS. ${ }^{11}$ Il est donc clair qu'il faut trouver un analgésique non opioïde pour les amygdalectomies à l'avenir.

La sous-utilisation des AINS après une amygdalectomie est principalement due aux inquiétudes persistantes concernant les saignements. L'épargne plaquettaire des inhibiteurs de la COX-2 n'est plus à démontrer. ${ }^{12}$ De plus, si l'on analyse les recherches, on constate que même l'ibuprofène, un AINS non sélectif, n'augmente pas les saignements post-amygdalectomie et est sécuritaire. ${ }^{13}$ Une méta-analyse récente portant sur plus de 1747 enfants étaye cette opinion. ${ }^{14}$ En revanche le kétorolac, un agent bien moins sélectif de la COX-2, n'est pas sécuritaire. ${ }^{13}$ En effet, les directives d'amygdalectomie de l'Ontario pour 2013 préconisent l'utilisation de routine de l'ibuprofène lors d'une amygdalectomie mais déconseillent l'utilisation du kétorolac. ${ }^{15}$

Dans une étude récente comparant la morphine à l'ibuprofène après une amygdalectomie, les conclusions de Kelly et coll. sont très convaincantes concernant l'administration de routine de l'ibuprofène. ${ }^{16}$ Dans cette étude, 91 patients pédiatriques souffrant de troubles respiratoires du sommeil ont été randomisés à recevoir de l'ibuprofène ou de la morphine en période périopératoire et ont été monitorés par oxymétrie au cours de la nuit avant et après leur opération. Au cours de la première nuit postopératoire, la désaturation en oxygène s'est améliorée chez $68 \%$ des patients du groupe ibuprofène, comparativement à $14 \%$ seulement des patients du groupe morphine $(P<0,01)$. De plus, le nombre d'épisodes de désaturation par heure a diminué dans le groupe ibuprofène mais augmenté dans le groupe morphine-à tel point que l'étude a été interrompue de façon précoce par le Comité de surveillance et de suivi de l'étude. La durée moyenne d'utilisation de l'analgésique (morphine ou ibuprofène) était d'environ quatre jours dans les deux groupes. Les deux groupes ont également fait état de scores de douleur semblables malgré la dose relativement élevée de morphine prescrite $\left(0,2-0,5 \mathrm{mg} \cdot \mathrm{kg}^{-1}\right.$ po aux quatre heures au besoin). Aucune différence n'a été observée en matière de saignements postopératoires, mais l'étude manquait de puissance pour évaluer cette complication.

Nous ne connaissons pas le taux d'utilisation actuel de l'ibuprofène pour les amygdalectomies-mais il semble faible. Ce taux d'utilisation peut-il être amélioré? Le taux d'adoption de la dexaméthasone comme agent de routine, qui s'appuie sur les directives américaines fondées sur des données probantes concernant les nausées et vomissements postopératoires après une amygdalectomie, peut nous donner une idée des obstacles à franchir avant l'adoption d'un autre agent. En 2011, l'Académie américaine
d'otolaryngologie-Chirurgie de la tête et du cou (American Academy of Otolaryngology_Head and Neck Surgery) publiait des directives fondées sur des données probantes pour la prise en charge de l'adéno-amygdalectomie. ${ }^{13}$ Ces directives n'abordaient pas directement la question de l'analgésie postopératoire mais préconisaient l'utilisation routinière de dexaméthasone et étaient clairement opposées à l'utilisation routinière d'antibiotiques. Pourtant, dans un compte rendu portant sur 111813 amygdalectomies et adéno-amygdalectomies publié en 2015, Mahant et coll. rapportaient que l'utilisation de dexaméthasone n'avait que peu augmenté-de 74,6\% des interventions avant la publication des directives à $77,4 \%$ des interventions en $2013(P<0,001){ }^{2}$ Cela pourrait être considéré comme une bonne réponse aux directives mais, à des fins de comparaison, accepterions-nous une automobile fiable à $77,4 \%$ seulement? Il faut souligner qu'il s'agissait de statistiques agrégées pour tous les patients; le taux d'adoption par hôpital se situait entre 63,3 et $95,7 \%$ (en d'autres mots, un tiers des patients n'ont pas reçu de dexaméthasone du tout dans certains hôpitaux). À l'inverse, l'utilisation d'antibiotiques avait diminué (tel que recommandé) de façon bien plus significative, passant de 34,7 à $21,8 \%(P<0,001)$. Les auteurs avancent plusieurs raisons possibles pour la réticence à utiliser la dexaméthasone, notamment la croyance, discréditée mais persistante, fondée sur une étude de 2008 ayant fait l'objet de beaucoup de publicité, selon laquelle la dexaméthasone augmenterait les saignements postopératoires. ${ }^{17}$ Cette étude fait figure d'anomalie dans la littérature portant sur la dexaméthasone, et ses analyses statistiques ont depuis été considérablement remises en question. ${ }^{18} \mathrm{~L}$ 'ibuprofène et les autres AINS non sélectifs seront confrontés aux mêmes difficultés d'adoption s'il existe la moindre croyance-qu'elle soit avérée ou non-qu'il y a un risque de saignement.

À la lumière de cet exemple édifiant, et malgré les directives ${ }^{13,15}$ préconisant son utilisation, nous ne pouvons pas nous attendre à ce que l'ibuprofène soit adopté de façon universelle par les médecins dans un avenir proche. Son association avec les saignements postopératoires semble être enracinée bien trop profondément dans la conscience collective du milieu pédiatrique. Peut-être avons-nous besoin d'un nouveau départ, avec une nouvelle classe de médicaments analgésiques, tels que les inhibiteurs de la COX-2? Des recherches supplémentaires sont de mise. Toutefois, la complaisance des médecins ne devrait pas s'ajouter à la liste déjà trop longue des embûches à des soins analgésiques optimaux en pédiatrie.

Conflicts of interest None declared. 


\section{Conflit d'intérêt Aucun.}

\section{References}

1. Stewart DW, Ragg PG, Sheppard S, Chalkiadis GA. The severity and duration of postoperative pain and analgesia requirements in children after tonsillectomy, orchidopexy, or inguinal hernia repair. Paediatr Anaesth 2012; 22: 136-43.

2. Mahant S, Hall M, Ishman SL, et al. Association of national guidelines with tonsillectomy perioperative care and outcomes. Pediatrics 2015; 136: 53-60.

3. Li X, Zhou M, Xia Q, Li J. Parecoxib sodium reduces the need for opioids after tonsillectomy in children: a double-blind placebocontrolled randomized clinical trial. Can J Anesth 2016; 63: this issue. DOI: 10.1007/s12630-015-0560-3

4. Juni P, Nartey L, Reichenbach S, Sterchi R, Dieppe PA, Egger M. Risk of cardiovascular events and rofecoxib: cumulative metaanalysis. Lancet 2004; 364: 2021-9.

5. Murto K, Lamontagne $C$, McFaul C, et al. Celecoxib pharmacogenetics and pediatric adenotonsillectomy: a doubleblinded randomized controlled study. Can J Anesth 2015; 62: 785-97.

6. Stempak D, Gammon J, Klein J, Koren G, Baruchel S. Singledose and steady-state pharmacokinetics of celecoxib in children. Clin Pharmacol Ther 2002; 72: 490-7.

7. Krishnaswami S, Hutmacher MM, Robbins JL, Bello A, West C, Bloom BJ. Dosing celecoxib in pediatric patients with juvenile rheumatoid arthritis. J Clin Pharmacol 2012; 52: 1134-49.

8. Ramos SD, Mukerji S, Pine HS. Tonsillectomy and adenoidectomy. Pediatr Clin North Am 2013; 60: 793-807.

9. Brown KA, Laferriere A, Lakheeram I, Moss IR. Recurrent hypoxemia in children is associated with increased analgesic sensitivity to opiates. Anesthesiology 2006; 105: 665-9.
10. Cote CJ, Posner KL, Domino KB. Death or neurologic injury after tonsillectomy in children with a focus on obstructive sleep apnea: Houston, we have a problem! Anesth Analg 2014; 118: 1276-83.

11. Goldman JL, Baugh RF, Davies L, et al. Mortality and major morbidity after tonsillectomy: etiologic factors and strategies for prevention. Laryngoscope 2013; 123: 2544-53.

12. Leese PT, Hubbard RC, Karim A, Isakson PC, Yu SS, Geis GS. Effects of celecoxib, a novel cyclooxygenase-2 inhibitor, on platelet function in healthy adults: a randomized, controlled trial. J Clin Pharmacol 2000; 40: 124-32.

13. Baugh RF, Archer SM, Mitchell RB, et al. Clinical practice guideline: tonsillectomy in children. Otolaryngol Head Neck Surg 2011; 144(1 Suppl): S1-30.

14. Riggin L, Ramakrishna J, Sommer DD, Koren G. A 2013 updated systematic review \& meta-analysis of 36 randomized controlled trials; no apparent effects of non steroidal anti-inflammatory agents on the risk of bleeding after tonsillectomy. Clin Otolaryngol 2013; 38: 115-29.

15. Provincial Council for Maternal and Child Health and Ministry of Health and Long-Term Care. Quality-Based Procedures Clinical Handbook for Paediatric Tonsillectomy with and without Adenoidectomy. December 2013. Province of Ontario Ministry of Health and Long-Term Care. Available from URL: http://www.health.gov.on.ca/en/pro/programs/ecfa/docs/qbp_ tonsil.pdf (accessed November 2015).

16. Kelly LE, Sommer DD, Ramakrishna J, et al. Morphine or ibuprofen for post-tonsillectomy analgesia: a randomized trial. Pediatrics 2015; 135: 307-13.

17. Czarnetzki C, Elia N, Lysakowski C, et al. Dexamethasone and risk of nausea and vomiting and postoperative bleeding after tonsillectomy in children: a randomized trial. JAMA 2008; 300: 2621-30.

18. Keller M, Brigger MT. The steroid controversy: where are we? ORL J Otorhinolaryngol Relat Spec 2013; 75: 155-64. 